\title{
Prevalence of dyslipidemia screened by Accutrend GCT
}

N.Fafa, D.Meskine, S.Fedala, L.Kedad

Hospital public establishment Bologhine Ibn Ziri

\section{Introduction :}

Obesity and especially abdominal obesity is therefore often associated with atherogenic dyslipidemia wich lead to cardiovascular complications.

The aim of our study was to analyze the distribution of cholesterol and triglycerids and to determine the prevalence of dyslipidemia in the Algerian adult population and their variation depending on the presence or absence of obesity .

\section{Methodology:}

Capillary samples using a validated lipids player (Accutrend GCT) were performed in 2210 individuals randomly selected from the general population. High cholesterol is defined by a fasting cholesterol (fasting 12 hours) $>2 \mathrm{~g} / \mathrm{I}$. The hypertriglyceridemia is defined by a triglyceride levels after 12 hours of fasting $>1.5 \mathrm{~g} / \mathrm{I}$, or a person treated to dyslipidemia.

\section{Results :}

The mean fasting total cholesterol in our sample was $1.70 \mathrm{~g} / \mathrm{I}$ (1 to 2.4)

-The Average fasting triglycerids is $1.15 \mathrm{~g} / \mathrm{I}$ (0.39 to 2.69).

-A known history of dyslipidemia was rated at $7.06 \%$ of subjects.

-A Dyslipidemia was detected in $26.11 \%$ of the individuals in our population
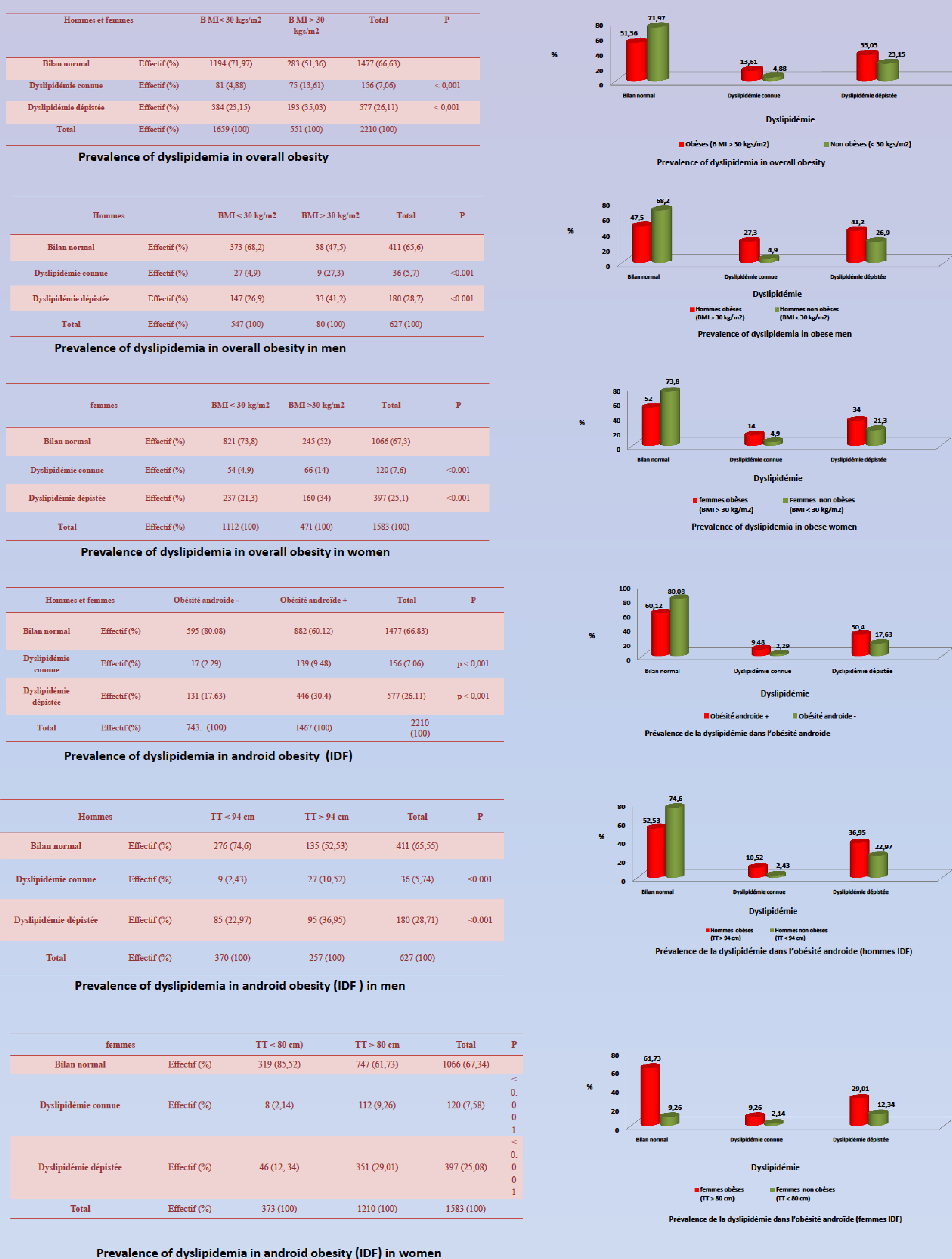

When looking at individuals with a body mass index above $30 \mathrm{~kg} / \mathrm{m} 2$, we note a prevalence of $13.61 \%$ of known dyslipidemia and $35.03 \%$ of dyslipidemia discovered during our investigation.

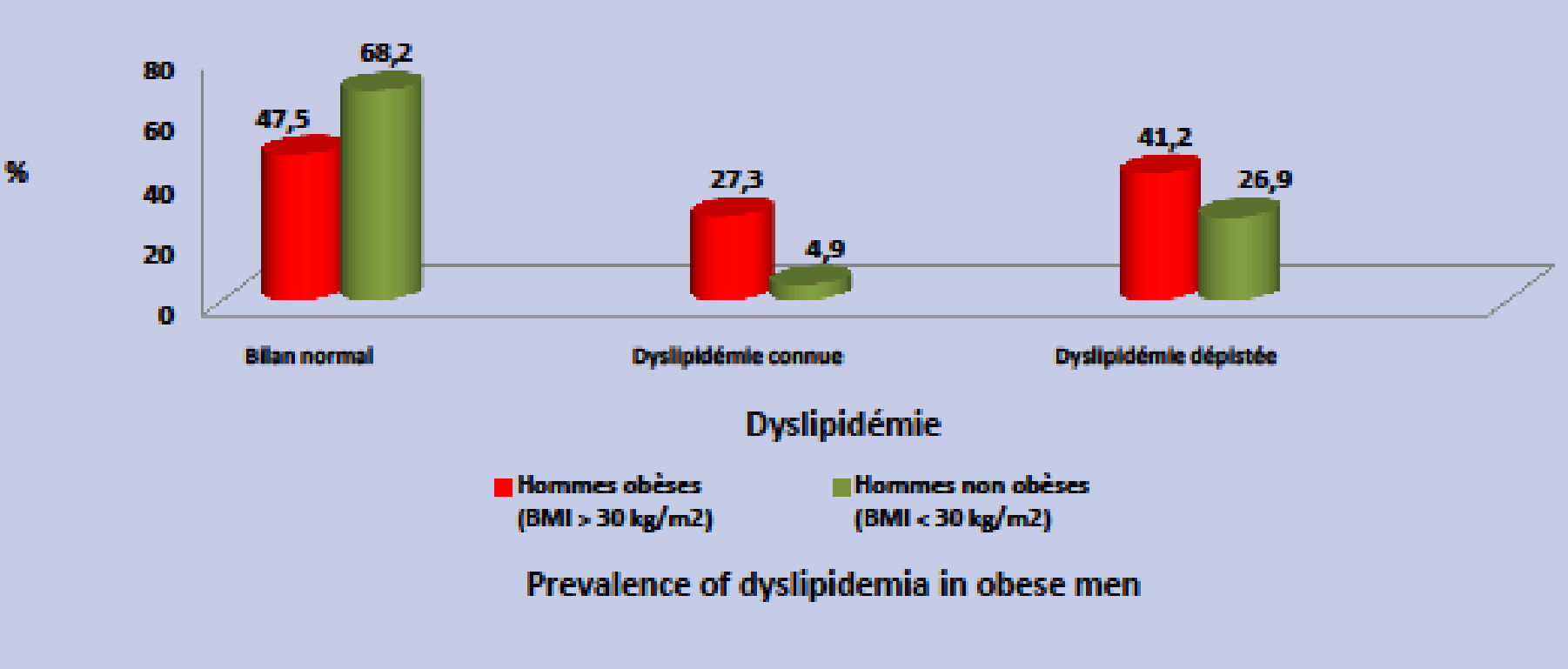

Among men the prevalence of detected dyslipidemia $41.2 \%$ in obese versus $26.9 \%$ in nonobese $(<0.0001)$ and $27.3 \%$ known dyslipidemia in obese versus $4.9 \%$ in non-obese ( $<0.0001)$

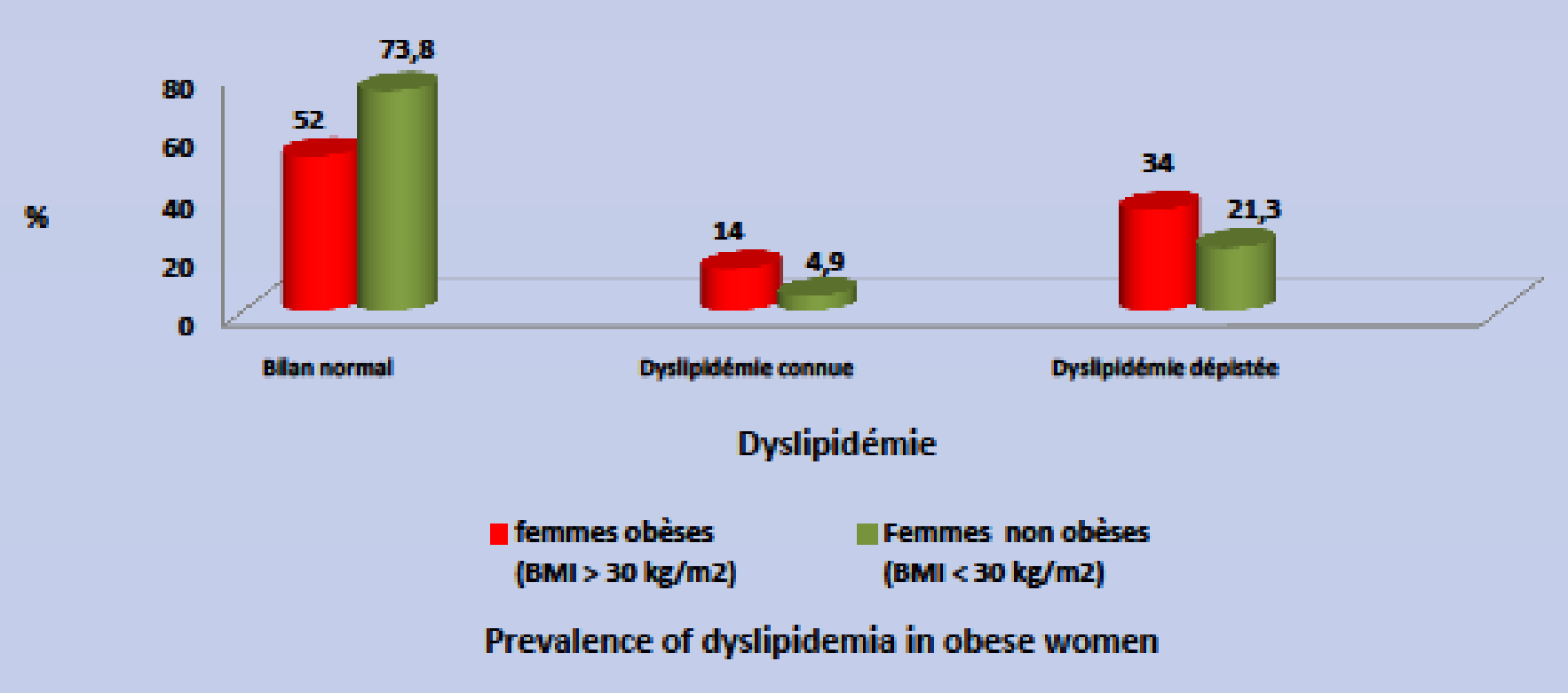

In women the prevalence of diagnosed dyslipidemia is denoted in $34 \%$ of obese versus
$21.3 \%$ of non-obese $(<0.0001)$ and dyslipidemia known in $14 \%$ of obese versus $4.9 \%$ of non- obese $(<0,0001)$.

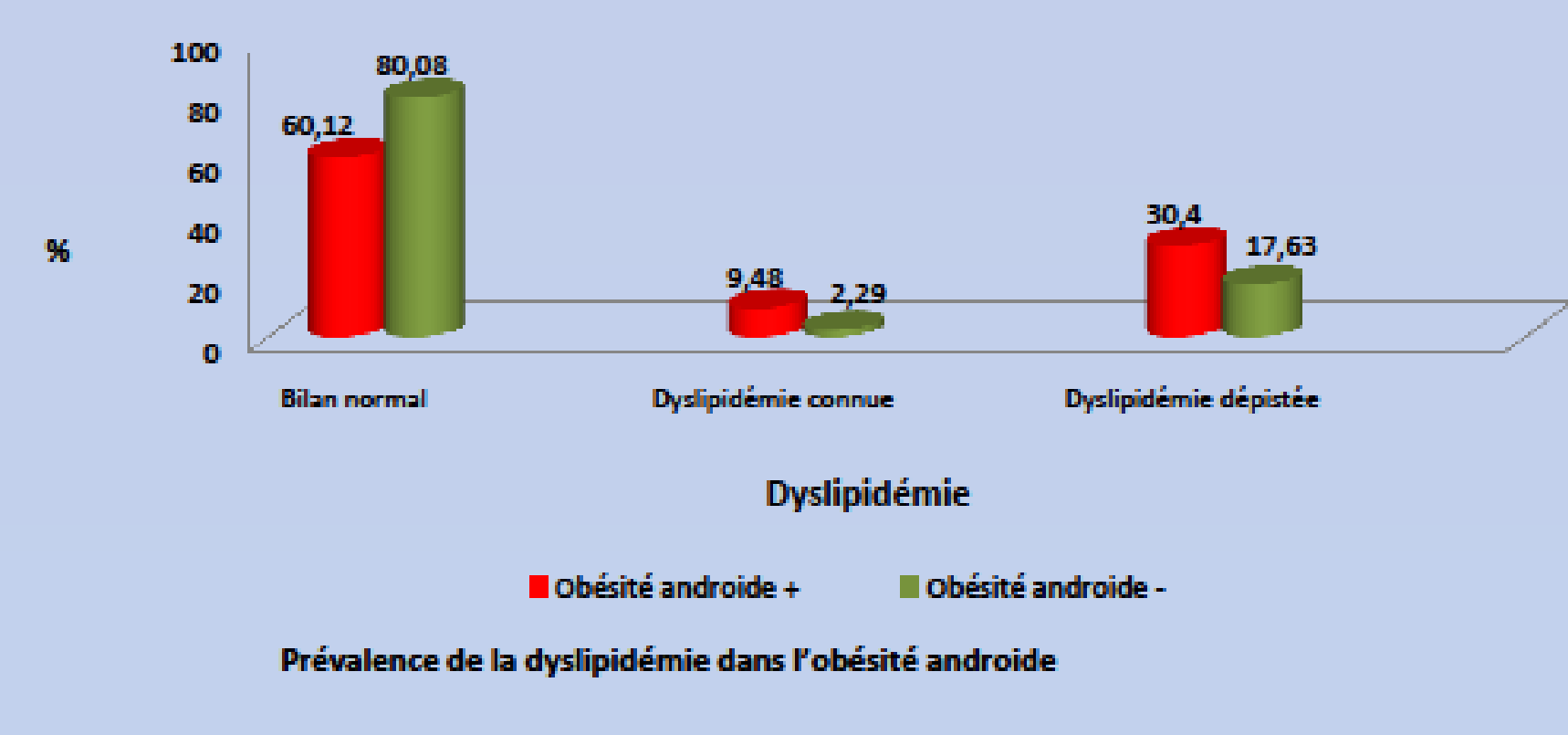

For abdominal obesity (IDF ) we find a prevalence of detected dyslipidemia $30.04 \%$ of obese versus $17.63 \%$ in non-obese $(<0.0001$ ) and the known dyslipidemia $9.48 \%$ in obese versus $2.29 \%$ in non-obese $(<0.0001)$

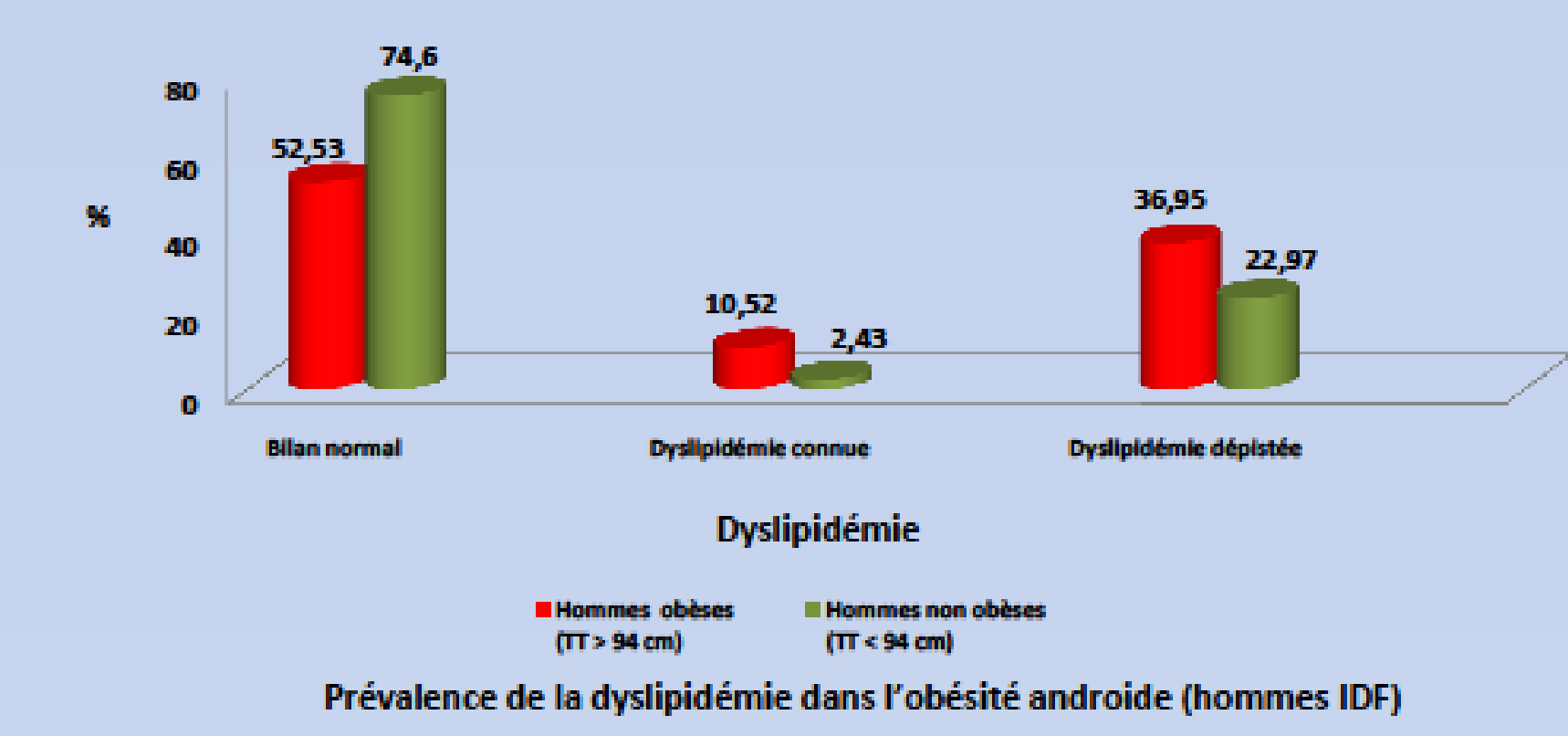

Among men the prevalence of detected dyslipidemia $36.95 \%$ of obese versus 22.97 in non-obese $(<0.0001$ ) and $10.52 \%$ of known dyslipidemia in obese versus $2.43 \%$ in nonobese $(<0.0001)$

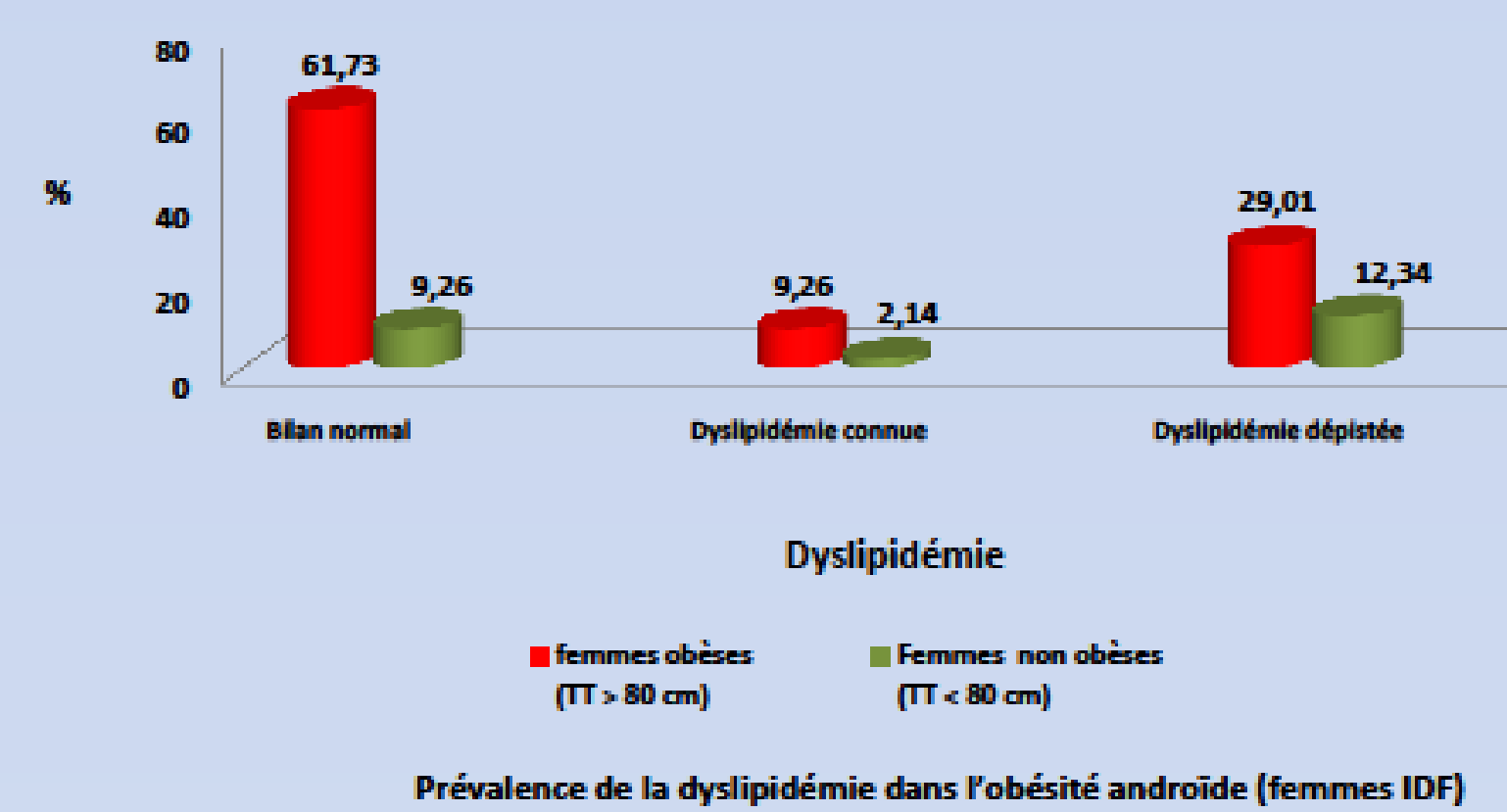

In women there is a prevalence of detected dyslipidemia $29.01 \%$ among those with abdominal obesity versus $12.34 \%$ in non-obese $(<0.0001)$ and the known dyslipidemia $9.26 \%$ in obese versus $2.14 \%$ in non-obese $(<0.001)$

\section{Discussion :}

In multivariate analysis, the known and diagnosed dyslipidemics are more obese than the normolipidemics :

OR1 known 2,4 (IC $95 \%$ 1,59-3,63) p < 0,001. OR2 diagnosed1,63 (IC 95 \% 1,27-2,08) p $<0,001$.

When looking at the android obesity (IDF ), known and tracked hyperlipidemic are also more obese than subjects with normal lipid profile OR1 known 2,27(IC 95\% 1,24-4,15) p < 0,001. OR2 detected 1,8 (IC 95\% 1,38-2,62) p $<0,001$.

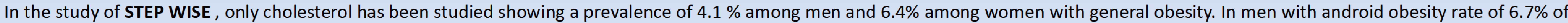
cholesterol was found versus $6.3 \%$ for women.

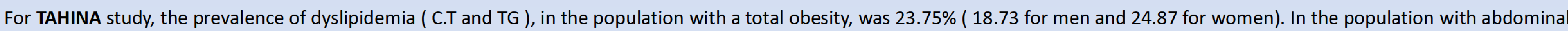
obesity (IDF) , the prevalence was $19.18 \%$ ( $19.07 \%$ versus $19.23 \%$ men women ), it was $21.77 \%$ ( $21.55 \%$ of men and $21,81 \%$ of women) when the NCEP -ATP III criteria are used

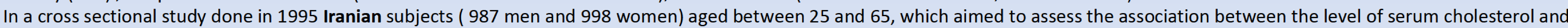
$\mathrm{BMI}$, noted an association positive between BMI and total cholesterol.

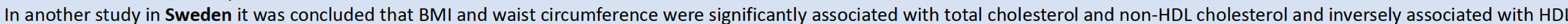
cholesterol levels.

A study among white American-born women (314) found that changes in BMI of $21.1 \mathrm{~kg} / \mathrm{m} 2$ and $30 \mathrm{~kg} / \mathrm{m} 2$ were associated with increased cholesterol level of $23 \mathrm{mg} / \mathrm{dl}$.

A work done among Turkish men , found a relationship between dyslipidemia, BMI , waist circumference and age.

\section{Conclusion :}

Dyslipidemia is a frequent complication of obesity that must be screened to prevent installatation of cardiovascular complications that often have implications for high morbidity and mortality and a significant financial cost. 